\section{Fatores relacionados à vacinação contra a gripe em idosos: estudo transversal, Cambé, Paraná, Brasil}

\author{
Factors associated with influenza vaccination \\ among the elderly: a cross-sectional study \\ in Cambé, Paraná State, Brazil
}

Eliane Cristina Campos 1 Luci Cristina Pulga Sudan 1 Edlivia Dias de Mattos 1 Reginaldo Fidelis 2

\footnotetext{
1 Universidade Norte do Paraná, Londrina, Brasil. 2 Universidade Tecnológica Federal do Paraná, Curitiba, Brasil.

Correspondência E. C. Campos

Universidade Norte do Paraná. Rua Charles Robert Darwim 65, Londrina, PR 86060-130, Brasil. elianecampos@hotmail.com
}

\begin{abstract}
The aim of this study was to assess influenza immunization coverage in the elderly and the association between vaccination and socioeconomic, demographic, health, and lifestyle variables, using a descriptive cross-sectional epidemiological approach from April 2008 to September 2009 with 990 elderly in Cambé, Paraná State, Brazil. Vaccination coverage was $74.6 \%$. The highest vaccination rate was among individuals 70 to 79 years of age [adjusted prevalence ratio $(P R)=$ 1.05] and 80 or older $(P R=1.03)$, with hypertension, and with monthly income up to 3 times the minimum wage $(P R=1.10)$ or higher $(P R=1.17)$ compared to elderly without income. Sedentary elderly $(P R=0.96)$ adhered less to vaccination, and divorced/separated individuals $(P R=0.92)$ had lower adherence compared to the married. The results suggest the need for healthcare strategies considering the factors that affect voluntary vaccination in order to help increase the odds of success for immunization programs.
\end{abstract}

Influenza Vaccines; Immunization Programs; Health of the Elderly

\section{Introdução}

Os idosos do Brasil somam aproximadamente 21 milhões de pessoas, representando $11 \%$ da população total (Instituto Brasileiro de Geografia e Estatística. http://www.censo2010.ibge.gov.br/si nopse/webservice/frm_piramide.php?codigo $=0$, acessado em 05/Nov/2010) e, conforme projeções da Organização das Nações Unidas, estima-se que em 2050 representarão 23,6\% da população e o Brasil será um dos cinco países com maior contingente de idosos do mundo 1 . Essa realidade demanda estratégias, ações e serviços que devem ser adotados para minimizar a ocorrência de agravos à saúde e prevenir as complicações de condições crônicas já existentes 2.

A população de idosos possui maior risco de ser acometida por doenças respiratórias infecciosas, e a gripe tem grande importância epidemiológica pela rápida evolução e potencial para complicações, como as pneumonias, amplamente associadas ao aumento da mortalidade desta população 3,4 , em especial nos grupos acometidos por doenças crônicas como insuficiência cardíaca, doenças pulmonares e diabetes 5 .

Segundo o Ministério da Saúde, estudos epidemiológicos demonstram que $75 \%$ das infecções respiratórias em idosos ocorrem por vírus Influenzae e, do total de internações por influenza e pneumonias em 2009, $24 \%$ foram em maiores de 60 anos 6 . Considerando o envelhecimento da 
população mundial, essa situação pode se agravar e requer ações efetivas de prevenção, controle e tratamento, representando um sério desafio da saúde pública na atualidade.

Nesse sentido, a Organização Mundial da Saúde (OMS) tem indicado desde a década de 1960 um programa de imunização para idosos em todo o mundo. O Brasil iniciou as campanhas nacionais de vacinação contra a gripe em 1999, abrangendo proteção específica não somente para influenza, mas também para pneumonia pneumocócica e tétano. Inicialmente, a vacina contra influenza foi recomendada a partir de 65 anos e, um ano mais tarde, em 2000, passou a ser oferecida a todos os idosos com 60 ou mais anos de idade e para a população indígena ${ }^{4,7}$. Em 2011, foi ampliada para três novos grupos: crianças de seis meses a menores de dois anos, gestantes e trabalhadores da saúde ${ }^{6}$.

Desde o início das campanhas de imunização tem sido constatada uma redução importante do número de casos de gripe entre idosos, principalmente para as regiões Sul, Centro-oeste e Sudeste 8 . Francisco et al. 9 demonstraram tendência para a queda da mortalidade por doenças respiratórias entre idosos brasileiros após a intervenção vacinal no período entre os anos de 1980 a 2000. Verificou-se ainda que a vacinação reduziu de $32 \%$ a $45 \%$ o número de hospitalizações por pneumonias e de $39 \%$ a $75 \%$ a mortalidade global ${ }^{8}$.

Até 2007, a meta mínima para cobertura vacinal estabelecida pelo Programa Nacional de Imunização (PNI) era de $70 \%$ da população alvo. Em 2008, foi ampliada para $80 \% 8$.

Ao longo dos últimos anos, a cobertura vacinal tem sido crescente e até superada a meta estimada em algumas regiões do país. Segundo o Ministério da Saúde, mais de 14 milhões de idosos foram imunizados em 2008, o que representa uma cobertura de $87 \%$ da população e, em 2009, foram imunizados mais de 15 milhões de idosos 8 . Reduzir o número de casos de gripe e suas complicações, as internações hospitalares e as mortes dos indivíduos mais vulneráveis são os objetivos da Campanha de Vacinação do Idoso $10,11,12$. Em sua 13a edição, em 2011, estimase que 19,4 milhões de idosos serão imunizados pelo SUS 6 .

No entanto, apesar dos avanços alcançados com as campanhas, as coberturas ainda têm sido insatisfatórias em vários municípios brasileiros que apresentam percentuais inferiores aos $80 \%$ recomendados pelo $\mathrm{PNI}$ 8,13,14.

Pesquisas demonstram que a adesão à vacinação é influenciada por fatores contextuais e individuais segundo grupos específicos e locais de residência 14 . Dentre esses fatores, tem-se ob- servado associação positiva entre a vacinação contra a gripe e a idade mais avançada, ou seja, idosos mais velhos aderem mais à vacinação comparados aos idosos na faixa etária de 60-69 anos 3,5,13,15,16. Em vários estudos constata-se a associação de variáveis como estado civil, escolaridade e classe econômica, bem como a presença de doenças crônicas (hipertensão arterial), de hábitos saudáveis (prática regular de exercícios físicos, não fumar) e o uso de serviços de saúde (consultas médicas recentes) com a adesão à vacinação 3,5,14,15,16. Sugere-se ainda que a não adesão é justificada, pelo menos em parte, por mitos relacionados aos eventos adversos pós-vacinais, a crença de que a gripe é uma doença banal e a falta de credibilidade na vacina $17,18,19$.

Considerando que esses fatores podem variar entre as populações, identificá-los e avaliar situações locais tornam-se fundamentais para orientar estratégias de intervenção efetivas, visando ao aumento da cobertura vacinal 3,14. De modo mais abrangente, sinaliza aspectos relevantes para a construção de políticas públicas e planos de ação voltados às necessidades locais em prol de suprir carências específicas. Fortalece as ações de promoção em saúde voltadas ao idoso, impactando diretamente na redução do número de internações hospitalares e da taxa de mortalidade associadas às complicações advindas da gripe, e ainda diminui a demanda por consultas médicas nos serviços de saúde. Dessa forma, além de melhorar a qualidade de vida dessa população, norteia a otimização dos gastos públicos com a assistência à saúde.

Nesse sentido, o presente estudo teve como objetivo estimar a cobertura vacinal contra a gripe em idosos residentes nas áreas de abrangência do Estratégia Saúde da Família (ESF) do Município de Cambé, Paraná, bem como verificar sua associação com as variáveis socioeconômicas, demográficas, de saúde e estilo de vida. Conhecer os fatores que interferem na não-adesão à vacinação pela população idosa também permitirá discutir novos desafios a serem superados pelo Programa Saúde da Família (PSF), enquanto principal estratégia de organização da atenção básica em saúde do Brasil.

\section{Métodos}

Estudo de natureza epidemiológico-descritiva, transversal, realizado por meio de inquérito domiciliar nos anos de 2008 e 2009 no Município de Cambé, localizado no norte do Estado do Paraná.

Nesta pesquisa foram considerados os dados do Sistema de Informação da Atenção Básica 
(SIAB) 2008/2009 (Departamento de Atenção Básica. http://dab.saude.gov.br/histori co_cobertura_sf_relatorio.php, acessado em 24/ Nov/2011), nos quais a estimativa da população total de Cambé era de 96.555 indivíduos, sendo $8.304(8,6 \%)$ com 60 anos ou mais de idade. A estimativa da população coberta pelo ESF era de 82.800 pessoas e a proporção de cobertura populacional estimada de $85,7 \%$. Segundo dados do IBGE, em 2010 a população do município era de 96.733 habitantes e destes, aproximadamente 11,7\% são idosos (Instituto Brasileiro de Geografia e Estatística. http://www.censo2010.ibge.gov. $\mathrm{br} /$ sinopse/webservice/frm_piramide.php?ano $=2010 \&$ codigo $=410370 \&$ corhomem $=88 \mathrm{C} 2 \mathrm{E} 6 \& \mathrm{c}$ ormulher $=$ F9C189\&wmaxbarra $=180$, acessado em 10/Out/2011).

A pesquisa, autorizada pela Secretaria de Saúde, foi desenvolvida nas áreas de abrangência das equipes do ESF das 11 unidades básicas de saúde (UBS) do município, após parecer favorável do Comitê de Ética da Universidade Norte do Paraná (UNOPAR), protocolo no. PP/0086/07.

A amostra de 990 indivíduos foi determinada estatisticamente a partir de uma população de 8.300 idosos do município. Utilizou-se a proporção de $50 \%$ para idosos dos sexos masculino e feminino, com o intuito de obter a amostra máxima. A amostra calculada foi de 367 pessoas, considerando erro máximo de $5 \%$ e, em virtude das possíveis perdas amostrais e da variação populacional no período de condução da pesquisa, foi elevada para 990 indivíduos. A amostra foi estratificada considerando-se o percentual de idosos cadastrados no PSF de UBS do município, segundo o sexo, devido as diferenças de densidade populacional.

A escolha dos participantes foi aleatória, por meio de sorteio com base nas fichas cadastrais dos idosos fornecidas pelos agentes comunitários de saúde (ACS), conforme as microáreas de abrangência. A coleta de dados foi realizada em entrevistas domiciliares acompanhadas pelos ACS e contou com a participação de 990 idosos com idades iguais ou superiores a 60 anos, de ambos os sexos, não institucionalizados e que aceitaram participar voluntariamente deste trabalho mediante assinatura prévia de Termo de Consentimento Livre e Esclarecido. Os idosos com alteração cognitiva puderam participar do estudo sendo representados por seus cuidadores. A avaliação da capacidade cognitiva foi realizada mediante informações obtidas em entrevista com o cuidador, tendo como critérios as respostas relacionadas à capacidade de linguagem e memória do idoso e diagnósticos médicos de Alzheimer, demência e alterações psiquiátricas.
As informações socioeconômicas e demográficas (sexo, idade, escolaridade, estado conjugal, arranjo familiar, renda pessoal mensal em salários mínimos), bem como as condições de saúde (autopercepção da saúde, doenças referidas, comorbidades, uso de medicamentos) e estilos de vida relacionados à saúde (prática de atividade física e tabagismo) foram avaliadas por meio de entrevista estruturada, sendo realizado o treinamento da equipe de pesquisadores de campo e pré-teste.

No presente trabalho, a variável dependente foi a vacinação contra gripe nos últimos 12 meses que antecederam a pesquisa, cuja informação foi obtida pela pergunta: "Nos últimos 12 meses você tomou vacina contra a gripe?”, amplamente adotada em estudos de fatores associados à vacinação porque apresenta boa sensibilidade (98\%), embora a especificidade seja mais baixa $(71 \%) 20$.

Na metodologia estatística, por meio dos testes de qui-quadrado de Pearson, exato de Fisher e teste G, analisou-se a homogeneidade da cobertura vacinal sendo considerado nível de significância de $5 \%(p<0,05)$. Foi estudada a associação entre vacinação contra a gripe e covariáveis de interesse, baseando-se na razão de prevalência (RP) e intervalos de 95\% de confiança (IC95\%), usando-se a regressão de Poisson bivariada e multivariada 21,22 . Todas as variáveis que apresentaram associação com a variável dependente na análise bivariada foram incluídas no modelo multivariado final, calculando-se razões de prevalência ajustadas. Os dados foram gerenciados utilizando-se os softwares estatísticos Epi Info versão 3.3.2 (Centers for Disease Control and Prevention, Atlanta, Estados Unidos), BioEstat versão 5.0 (Sociedade Civil Mamirauá, Manaus, Brasil) e SPSS versão 17 (SPSS Inc., Chicago, Estados Unidos).

\section{Resultados}

Dos 990 participantes, $42,7 \%$ eram homens e $57,3 \%$ mulheres, com média de idades igual a 71,9 (desvio-padrão - DP = 8,0). A faixa etária predominante foi de idosos jovens, de 60-69 anos $(43,8 \%)$, seguida de idosos de 70 a 79 anos $(38,2 \%)$. Os idosos mais velhos, com 80 ou mais anos de idade, representaram $18 \%$ da amostra. A cobertura vacinal contra a gripe neste estudo foi de 74,7\% (IC95\%: 71,9-77,4).

A Tabela 1 apresenta os resultados da análise simples da associação entre a vacinação contra a gripe e características sociodemográficas. Nessa análise, RP estatisticamente significantes foram verificadas para faixa etária (70-79 anos e $\geq 80$ 
Análise não ajustada da associação entre características sociodemográficas e vacinação contra a gripe em idosos. Município de Cambé, Paraná, Brasil, 2009.

\begin{tabular}{|c|c|c|c|c|c|c|}
\hline & \multicolumn{6}{|c|}{ Vacinação } \\
\hline & Sim & Não & Total & $\%$ vacinada (IC95\%) & RP (IC95\%) & Valor de p (5\%) \\
\hline Gênero & & & & & & 0,130 * \\
\hline Feminino & 413 & 154 & 567 & $72,9(69,2-76,5)$ & 1,00 & \\
\hline Masculino & 326 & 97 & 423 & $77,1(73,1-81,1)$ & $1,06(0,98-1,14)$ & 0,075 \\
\hline Faixa etária (anos) & & & & & & 0,002 * \\
\hline $60-69$ & 301 & 133 & 434 & $69,4(65,0-73,7)$ & 1,00 & \\
\hline $70-79$ & 302 & 76 & 378 & $79,9(75,9-83,9)$ & $1,15(1,06-1,25)$ & $\leq 0,001$ * \\
\hline 80 ou + & 136 & 42 & 178 & $76,4(70,2-82,6)$ & $1,10(0,99-1,22)$ & 0,049 * \\
\hline Total & 739 & 251 & 990 & $74,7(71,9-77,4)$ & & \\
\hline Cor & & & & & & 0,369 * \\
\hline Branca & 571 & 192 & 763 & $74,9(71,8-77,9)$ & 1,00 & \\
\hline Amarela & 12 & 1 & 13 & $92,3(77,8-100,0)$ & $1,23(1,05-1,45)$ & 0,130 \\
\hline Parda & 88 & 34 & 122 & $72,2(64,2-80,1)$ & $0,96(0,86-1,08)$ & 0,300 \\
\hline Preta & 68 & 24 & 92 & $73,9(64,9-82,9)$ & $0,99(0,87-1,12)$ & 0,474 \\
\hline Estado civil & & & & & & $0,169 * \star$ \\
\hline Casado & 460 & 145 & 605 & $76,0(72,6-79,4)$ & 1,00 & \\
\hline Divorciado/Separado & 34 & 21 & 55 & $61,8(49,0-74,7)$ & $0,81(0,66-1,01)$ & $0,015 * *$ \\
\hline Solteiro & 23 & 12 & 35 & $65,7(50,0-81,4)$ & $0,86(0,68-1,10)$ & 0,119 \\
\hline Viúvo & 213 & 70 & 283 & $75,3(70,2-80,3)$ & $0,99(0,91-1,07)$ & 0,434 \\
\hline Não informado & 9 & 3 & 12 & $75,0(50,5-99,5)$ & $0,99(0,71-1,37)$ & 0,398 \\
\hline Anos de estudos & & & & & & $0,375 * *$ \\
\hline $1-3$ & 305 & 91 & 396 & $77,0(72,9-81,2)$ & 1,00 & \\
\hline $4-7$ & 108 & 44 & 152 & $71,1(63,8-78,3)$ & $0,92(0,82-1,03)$ & 0,090 \\
\hline $8-11$ & 21 & 11 & 32 & $65,7(49,2-82,1)$ & $0,85(0,66-1,10)$ & 0,107 \\
\hline $12 \mathrm{ou}+$ & 9 & 5 & 14 & $64,3(39,2-89,4)$ & $0,83(0,56-1,24)$ & 0,216 \\
\hline Analfabeto & 293 & 100 & 393 & $74,6(70,2-78,9)$ & $0,97(0,89-1,05)$ & 0,234 \\
\hline Ignorado & 3 & 0 & 3 & $100,0(-)$ & & \\
\hline 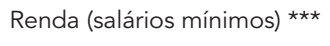 & & & & & & $0,003 * \star$ \\
\hline Não tem renda & 25 & 17 & 42 & $59,5(44,7-74,4)$ & 1,00 & 0,031 ** \\
\hline Até 3 & 689 & 230 & 919 & $75,0(72,2-77,8)$ & $1,26(0,98-1,62)$ & 0,019 * \\
\hline Acima de 3 & 25 & 4 & 29 & $86,2(74,7-98,8)$ & $1,45(1,09-1,93)$ & 0,015 * \\
\hline Moradia & & & & & & 0,762 * \\
\hline Acompanhado & 653 & 220 & 873 & $74,8(71,9-77,7)$ & 1,00 & \\
\hline Sozinho & 86 & 31 & 117 & $73,5(65,5-81,5)$ & $0,98(0,88-1,10)$ & 0,425 \\
\hline
\end{tabular}

IC95\%: intervalo de 95\% de confiança; RP: razão de prevalência não ajustada.

* Teste qui-quadrado significativo: $p<0,05$

** Teste G significativo: $p<0,05$;

*** Salário mínino para aposentados e pensionistas: R\$ 510,00 (2010).

anos), estado conjugal (casado e separado/divorciado) e renda pessoal mensal, considerando o nível de significância de 0,05.

Com relação à idade, maior cobertura vacinal foi verificada entre indivíduos na faixa etária de $70-79$ anos $(79,9 \%)$ e de 80 ou mais anos de idade (76,4\%), comparados aos idosos de 60-69 anos $(69,4 \%)$, com associação estatística significativa
( $p=0,002)$. As RP foram de 1,15 pessoa na faixa etária de 70-79 anos vacinada e 1,10 idoso com 80 ou mais anos vacinado para cada pessoa com idades de 60-69 anos vacinada ( $\mathrm{p}<0,05)$.

Não foi evidenciada diferença estatística entre vacinação e sexo ( $p=0,13)$, ambos apresentando padrão semelhante de distribuição percentual para a vacinação. 
Verificou-se que não existe dependência entre a variável estado civil e vacinação $(p>0,05)$. Entretanto, o estado civil separado/divorciado teve uma associação negativa com a adesão à vacinação (RP = 0,81; IC95\%: 0,66-1,01) comparado aos idosos casados $(\mathrm{p}<0,05)$.

Este estudo mostrou dependência entre a variável renda e a vacinação, pois entre os idosos que não possuíam renda mensal verificou-se que $59,5 \%$ tomaram a vacina, mas os maiores percentuais foram para aqueles com renda de até 3 salários mínimos (SM) (75\%) ou superior a 3 salários $(86,2 \%)$. A frequência das pessoas vacinadas com renda de até $3 \mathrm{SM}$ é 1,26 (RP) vez superior a das pessoas vacinadas sem renda mensal. Já a frequência das pessoas vacinadas com renda superior a 3 SM corresponde a 1,45 (RP) vez a das pessoas vacinadas sem renda mensal. As RP foram estatisticamente significantes $(\mathrm{p}=0,01)$.

Os resultados da análise bivariada dos indicadores de saúde e estilo de vida associados à vacinação estão apresentados na Tabela 2. Razões de prevalência estatisticamente significantes ( $p$ $<0,05$ ) foram observadas para doenças crônicas autorreferidas, relato de hipertensão arterial e realização de atividade física.

Na presente análise, constatou-se associação positiva entre a presença de doença crônica, hipertensão arterial e a vacinação $(p<0,01)$. Entre as doenças crônicas mais referidas pelos idosos figuraram: a hipertensão arterial (66,5\%), as doenças osteomioarticulares (28\%), diabetes (20,8\%), cardiopatias $(16,9 \%)$ e as pneumopatias $(6,4 \%)$. A associação entre o relato de doenças crônicas e a hipertensão arterial foi significativa ( $\mathrm{p} \leq 0,001$ ). A análise das RP entre cada doença e a vacinação contra a gripe foi estatisticamente significativa somente para a hipertensão arterial, doença prevalente entre idosos. Os idosos que referiram as demais doenças mencionadas apresentaram cobertura vacinal semelhante àqueles que não as referiram.

Em relação às comorbidades não foi encontrada associação estatística significativa entre a presença de comorbidades referidas pelos idosos e a vacinação.

Ao analisar os dados referentes ao estilo de vida, este trabalho verificou associação positiva entre prática de atividades físicas e a vacinação contra a gripe $(\mathrm{p}<0,05)$. Portanto, o sedentarismo apresentou associação negativa com o comparecimento à vacinação (RP = 0,92; IC95\%: 0,860,99). Já em relação ao tabagismo e a vacinação não houve associação estatística significativa entre as variáveis.

A Tabela 3 mostra os resultados finais da análise multivariada de regressão de Poisson dos fatores associados à vacinação contra a gripe. A faixa etária 70-79 anos, possuir renda mensal e maior renda, ter diagnóstico de hipertensão arterial e realizar atividades físicas regularmente, estiveram independentemente associadas ao comparecimento à vacinação. Associação negativa e significante foi encontrada para o estado conjugal (divorciado/separado) $(\mathrm{RP}=0,92$; IC95\%: 0,85-0,99). Nesta análise, não foi confirmada a associação estatística entre a vacinação e a presença de doenças crônicas, com exceção da hipertensão arterial.

\section{Discussão}

Os resultados mostraram que a cobertura vacinal contra a gripe entre idosos do Município de Cambé no período de 2008 a 2009 (74,7\%; IC95\%: $71,9-77,4)$, esteve abaixo da meta de $80 \%$ estabelecida pelo Ministério da Saúde 23.

Outros estudos brasileiros obtiveram porcentagens variáveis e ainda menores de cobertura vacinal. Na pesquisa de Lima-Costa 3 realizada na Região Metropolitana de Belo Horizonte (Minas Gerais) em 2003, a prevalência da vacinação foi de $66,3 \%$. Percentual semelhante foi referido por Francisco et al. 15 em estudo de base populacional conduzido em seis municípios do Estado de São Paulo, com 66,1\% de adesão à vacinação no período de 2001 a 2002. Em outra investigação, em Campinas (São Paulo), no período de 2008 a 2009, a taxa de imunização foi de $62,6 \%$. Dip 5 verificou $73 \%$ de cobertura vacinal em idosos residentes na área de abrangência de uma UBS de Londrina (Paraná), cidade vizinha a Cambé.

Vários são os fatores apontados na literatura que contribuem para as baixas coberturas vacinais, entre eles, o descrédito sobre a eficácia da vacina, a preocupação com a ocorrência de efeitos adversos, a crença de que a gripe é uma doença banal e a vacinação não é necessária, e a falta de orientação dos profissionais da saúde $14,18,24,25$.

Embora um dos principais motivos alegados para a não-adesão seja o medo de eventos adversos, a prevalência destes eventos pós-vacinais é baixa. Quando presentes, restringem-se à dor no local da vacina e ao surgimento de um quadro gripal leve, com coriza, dor de garganta, tosse e, mais raramente, a febre. Além disso, não necessariamente esses sintomas são decorrentes da vacinação, uma vez que podem vir associados a uma grande diversidade de agentes virais, além do vírus Influenzae 5,13.

Tais aspectos reforçam a importância das práticas educativas, orientação clara quanto aos riscos e benefícios à saúde do idoso, e destaca o papel das equipes de saúde no sucesso da 
Análise não ajustada da associação entre indicadores das condições de saúde, estilo de vida e vacinação contra a gripe em idosos. Município de Cambé, Paraná, Brasil, 2009.

\begin{tabular}{|c|c|c|c|c|c|c|}
\hline & \multicolumn{6}{|c|}{ Vacinação } \\
\hline & Sim & Não & Total & \% vacinada (IC95\%) & RP (IC95\%) & Valo de p (5\%) \\
\hline Autopercepção da saúde & & & & & & 0,531 * \\
\hline Ótima & 73 & 31 & 104 & $70,2(61,4-79,0)$ & 1,00 & \\
\hline Boa & 318 & 113 & 431 & $73,8(69,6-77,9)$ & $1,05(0,92-1,21)$ & 0,268 \\
\hline Regular & 279 & 84 & 363 & $76,9(72,5-81,2)$ & $1,09(0,95-1,26)$ & 0,103 \\
\hline Ruim & 69 & 23 & 92 & $75,0(66,2-83,8)$ & $1,07(0,90-1,27)$ & 0,277 \\
\hline Hospitalizado no último ano & & & & & & 0,988 * \\
\hline $\operatorname{Sim}$ & 141 & 48 & 189 & $74,6(68,4-80,8)$ & 1,00 & \\
\hline Não & 598 & 203 & 801 & $74,7(71,6-77,7)$ & $1(0,91-1,10)$ & 0,469 \\
\hline Doenças crônicas autorreferidas & & & & & & 0,008 * \\
\hline Sim & 659 & 208 & 867 & $76,0(73,2-78,9)$ & 1,00 & \\
\hline Não & 80 & 43 & 123 & $65,1(56,6-73,5)$ & $0,86(0,75-0,98)$ & 0,006 * \\
\hline Hipertensão arterial & & & & & & $\leq 0,001 *$ \\
\hline Sim & 514 & 144 & 658 & $78,1(75,0-81,3)$ & 1,00 & \\
\hline Não & 225 & 107 & 332 & $67,8(62,7-72,8)$ & $0,87(0,80-0,94)$ & $\leq 0,001 *$ \\
\hline Doenças osteomioarticulares & & & & & & 0,239 * \\
\hline Sim & 214 & 63 & 277 & $77,3(72,3-82,2)$ & 1,00 & \\
\hline Não & 525 & 188 & 713 & $73,6(70,4-76,9)$ & $0,95(0,88-1,03)$ & 0,136 \\
\hline Diabetes & & & & & & 0,096 * \\
\hline Sim & 163 & 43 & 206 & $79,1(73,6-84,7)$ & 1,00 & \\
\hline Não & 576 & 208 & 784 & $73,5(70,4-76,6)$ & $0,93(0,86-1,01)$ & 0,058 \\
\hline Cardiopatias & & & & & & 0,152 * \\
\hline Sim & 132 & 35 & 167 & $79,0(72,9-85,2)$ & 1,00 & \\
\hline Não & 607 & 216 & 823 & $73,8(70,7-76,8)$ & $0,93(0,85-1,02)$ & 0,091 \\
\hline Pneumopatias & & & & & & 0,791 \\
\hline $\operatorname{Sim}$ & 50 & 13 & 63 & $79,4(69,4-89,4)$ & 1,00 & \\
\hline Não & 689 & 238 & 927 & $74,4(71,5-77,1)$ & $0,94(0,82-1,07)$ & 0,229 \\
\hline Comorbidades & & & & & & 0,229 * \\
\hline 1 doença & 181 & 64 & 245 & $73,9(68,4-79,4)$ & 1,00 & \\
\hline 2 doenças & 194 & 65 & 259 & $74,9(69,6-80,2)$ & $1,01(0,92-1,12)$ & 0,435 \\
\hline 3 doenças & 136 & 48 & 184 & $73,9(67,6-80,3)$ & $1,00(0,89-1,12)$ & 0,459 \\
\hline 4 doenças & 74 & 16 & 90 & $82,2(74,3-90,1)$ & $1,11(0,99-1,26)$ & 0,074 \\
\hline 5 ou mais & 74 & 15 & 89 & $83,2(75,4-90,9)$ & $1,13(1,00-1,27)$ & 0,053 \\
\hline Atividade física & & & & & & 0,034 * \\
\hline Sim & 294 & 81 & 375 & $78,4(74,2-82,6)$ & 1,00 & \\
\hline Não & 445 & 170 & 615 & $72,4(68,8-75,9)$ & $0,92(0,86-0,99)$ & 0,020 * \\
\hline Fumante & & & & & & $0,308 * \star$ \\
\hline Não & 650 & 217 & 867 & $75,0(72,1-77,9)$ & 1,00 & \\
\hline Sim & 86 & 34 & 120 & $71,7(63,6-79,7)$ & $0,96(0,85-1,08)$ & 0,252 \\
\hline Ex-fumante & 3 & 0 & 3 & 100,0 & 1,33 (1,28-1,39) & 0,37 \\
\hline
\end{tabular}

IC95\%: intervalo de 95\% de confiança; RP: razão de prevalência não ajustada.

* Teste qui-quadrado significativo: $p<0,05$

** Teste $G$ significativo: $p<0,05$;

*** Teste exato de Fisher significativo: $p<0,05$. 
Análise ajustada da associação dos fatores relacionados à vacinação contra a gripe em idosos. Município de Cambé, Paraná, Brasil, 2009.

\begin{tabular}{|c|c|c|}
\hline & RP (IC95\%) & Valor de $\mathrm{p}(5 \%)$ \\
\hline \multicolumn{3}{|l|}{ Faixa etária (anos) } \\
\hline $60-69$ & 1,00 & \\
\hline $70-79$ & $1,05(1,02-1,09)$ & 0,004 * \\
\hline $80 \mathrm{ou}+$ & $1,03(0,99-1,08)$ & 0,152 * \\
\hline \multicolumn{3}{|l|}{ Estado civil } \\
\hline Casado & 1,00 & \\
\hline Divorciado/Separado & $0,92(0,85-0,99)$ & 0,029 * \\
\hline Solteiro & $0,95(0,86-1,05)$ & 0,358 \\
\hline Viúvo & $0,99(0,95-1,02)$ & 0,422 \\
\hline Não informado & $0,99(0,85-1,14)$ & 0,838 \\
\hline \multicolumn{3}{|l|}{ Renda (salários mínimos) ** } \\
\hline Não tem renda & 1,00 & \\
\hline Até 3 & $1,10(1,00-1,21)$ & 0,041 * \\
\hline Acima de 3 & $1,17(1,04-1,32)$ & 0,007 * \\
\hline \multicolumn{3}{|c|}{ Doenças crônicas autorreferidas } \\
\hline Sim & 1,00 & \\
\hline Não & $0,98(0,92-1,04)$ & 0,444 \\
\hline \multicolumn{3}{|l|}{ Hipertensão arterial } \\
\hline Sim & 1,00 & \\
\hline Não & $0,96(0,92-1,00)$ & 0,034 * \\
\hline \multicolumn{3}{|l|}{ Atividade física } \\
\hline Sim & 1,00 & \\
\hline Não & $0,96(0,93-0,99)$ & 0,018 * \\
\hline
\end{tabular}

IC95\%: intervalo de 95\% de confiança; RP: razão de prevalência ajustada por meio da análise multivariada.

(Regressão de Poisson) para todas as variáveis que obtiveram nível de significância inferior a 0,05.

* Teste Wald significativo: $p<0,05$;

** Salário mínino para aposentados e pensionistas: R\$ 510,00 (2010).

vacinação 5,25, pois a orientação de profissionais é um dos fatores que tem influenciado positivamente na adesão à vacina 14 .

Neste trabalho, o sexo não apresentou associação estatística com a vacinação contra a gripe, resultado consistente com a maioria dos estudos 13,15,26. Contudo, a falta de diferença de cobertura vacinal entre homens e mulheres pode ser resultado da baixa procura do sexo masculino pelos serviços de atenção básica, onde as mulheres são reconhecidamente suas maiores usuárias. Tal fato não evidencia que os homens adoecem menos, já que os mesmos também são acometidos por patologias que podem se apresentar de forma severa e crônica, contribuindo para que o índice de mortalidade seja maior do que no sexo feminino 27 .

Segundo a literatura específica sobre o assunto, os fatores socioculturais influenciam direta- mente na baixa procura dos homens pelos serviços de saúde e a associação do cuidar ao âmbito feminino é uma das principais explicações. No imaginário social, o cuidado não é visto como uma prática masculina e os homens não reconhecem a importância da promoção da saúde e prevenção de doenças como questões associadas ao sexo masculino 27,28 .

Para muitos, a atitude de procurar por serviços de saúde representa assumir suas fragilidades, medos e inseguranças, o que coloca em risco o sentimento de invulnerabilidade, um dos eixos da masculinidade socialmente instituída 27.

Entretanto, há que se considerar o fato de os homens perceberem os serviços públicos como um espaço frequentado por mulheres. A forma de acolhimento, a abordagem e o profissional que presta atendimento, na maioria das vezes do sexo feminino, podem representar uma barreira 
de acesso ao serviço. Assim, a organização dos serviços de saúde não estimula o acesso masculino, é deficiente em absorver a demanda apresentada pelos homens, e as próprias campanhas de saúde pública não se voltam para este segmento, consequentemente, os homens não se reconhecem como alvo do atendimento de programas de saúde 27,28.

Outro fato que contribui para os homens não buscarem os serviços de saúde, principalmente na atenção básica, está relacionado aos horários de funcionamento, já que o homem atribui dificuldade de conciliar horário de trabalho com o atendimento estabelecido pelo serviço. Convém ressaltar que muitos idosos, mesmo aposentados, ainda estão inseridos no mercado de trabalho.

A maioria dos estudos realizados sobre a saúde do idoso assistido pelas equipes do ESF mostra a prevalência no atendimento às mulheres idosas, o que pode expressar a falta de atenção dos profissionais na inclusão de homens idosos no PSF.

O Ministério da Saúde, em 2009, criou a Política Nacional de Atenção Integral à Saúde do Homem com o objetivo de promover ações em saúde voltadas à população masculina adulta, direcionando mudanças nas estratégias dos serviços de saúde e no enfoque relacionado ao sexo masculino 29.

Cabe então, às equipes de saúde da família, maior conscientização, atenção e envolvimento nas ações de promoção da saúde e prevenção de agravos, além de discutir os vários aspectos que possam contribuir para o acesso facilitado do homem ao serviço de saúde, tendo como ferramenta imprescindível a educação em saúde. Dessa forma, o estabelecimento de vínculo, a sensibilização e a construção de comportamento preventivo irão propiciar a participação do homem de forma contínua e rotineira nos programas de promoção à saúde.

A idade tem sido descrita como um fator associado à vacinação contra a gripe. Neste estudo foi encontrada maior adesão de idosos com idade mais avançada, de 70 ou mais anos. Outros trabalhos brasileiros corroboram os resultados encontrados 5,14,30. Sugere-se que idosos jovens, na faixa etária de 60-69 anos, tendem a uma autopercepção positiva da própria saúde, avaliada como boa, por isto comparecem menos à vacinação 5,31 .

Não foi verificada diferença na vacinação de idosos segundo as variáveis socioeconômicas e demográficas: escolaridade, estado civil, cor da pele e moradia (sozinho ou acompanhado). Contudo, analisando a associação entre indivíduos casados e os divorciados/separados, constatou-se que os idosos casados aderiram mais à vacinação que os divorciados/separados, com associação estatística significativa entre os grupos. Lima-Costa ${ }^{3}$ encontrou associação negativa e independente para idosos solteiros na Região Metropolitana de Belo Horizonte.

Na presente análise, a renda pessoal mensal esteve associada à taxa de imunização e os idosos que declararam renda maior aderiram mais à vacinação, como também foi observado por Vilarino et al. ${ }^{30}$. Nas pesquisas realizadas por Dip 5, Donalisio et al. 13 e Francisco et al. 15 não foram verificadas a associação entre renda per capita $\mathrm{e}$ a vacinação.

Vilarino et al. 30 relatam que a população de idosos de menor poder aquisitivo e de maior vulnerabilidade social procura os serviços de saúde de atenção básica ao ficar doente, sugerindo que não percebe ou não valoriza a importância da vacinação. Sugere-se que tal comportamento esteja intrinsecamente influenciado pela fragilidade social, cultural e intelectual dessa população 32 . Já o grupo de maior renda, busca a vacinação no serviço público e quando se sente doente procura o serviço privado ou conveniado.

Nesse sentido, faz-se necessária uma abordagem mais realista da complexa problemática social da saúde, e a atuação dos profissionais deve se fundamentar tanto na orientação acessível, como é indispensável que atuem como incentivadores do autocuidado à saúde, buscando facilitar o entendimento e estimular a participação dos idosos de baixa renda nas ações de promoção da saúde ${ }^{32}$.

Não foi verificada associação da vacinação com as seguintes variáveis de saúde e estilo de vida: autopercepção da saúde, hospitalização no último ano, comorbidades associadas e tabagismo, o que sugere uma falha de critérios epidemiológicos direcionando as ações em saúde.

No presente estudo pode-se verificar maior prevalência de vacinação entre os portadores de doenças crônicas apenas na análise simples. Após análise ajustada, a prevalência de vacinação foi significativamente maior somente entre os idosos hipertensos. Pesquisas apontam que o fato dos idosos hipertensos estarem em maior contato com a UBS facilita o comparecimento às campanhas ${ }^{13}$. Resultados semelhantes foram obtidos por outros autores 3,14,15.

Outras doenças crônicas como diabetes, doenças osteomioarticulares, cardiopatias e doenças pulmonares, bem como a presença de comorbidades, além da referência de hospitalização no último ano, não determinaram a adesão à vacinação como era esperado para estes grupos de risco, merecendo maior atenção nas campanhas públicas. Cabe destacar que os benefícios da vacinação para portadores de 
doenças crônicas e comorbidades, na prevenção de hospitalizações e óbitos, indicam formalmente a imunização 13 .

Segundo Dip \& Cabrera ${ }^{33}$, os médicos ainda não incorporaram a prescrição da vacina à prática habitual, e apontam que os idosos que não aderiram à vacinação o teriam feito caso houvesse recomendação médica. Outro estudo encontrou maior adesão à vacina associada à indicação por parte da enfermagem 34 . Tais fatos implicam uma reflexão dos profissionais de saúde sobre a sua responsabilidade como promotores de saúde 33 .

Desse modo, toda a equipe de saúde incluindo a classe médica, os enfermeiros e os agentes comunitários devem estar mais envolvidos no esclarecimento dos diversos aspectos relacionados aos benefícios da vacina, assim como na motivação dos idosos, além de se conscientizarem quanto aos prejuízos associados à nãovacinação, e que a omissão nesse sentido pode comprometer a qualidade de vida da população idosa 33.

Também verificamos que a probabilidade de ser vacinado foi maior entre os idosos que realizavam algum tipo de atividade física regular, corroborando com os resultados encontrados em outras pesquisas $3,14,30$, e reflete a ideia de que a postura de autocuidado ou de preservação da própria saúde está intrinsecamente associada a um estilo de vida saudável. Da mesma forma, alguns estudos demonstram que a proporção de fumantes é bem mais baixa entre os vacinados 5 , fato não constatado na presente investigação.
A orientação à pessoa idosa sobre a importância da vacinação constitui um dos elementos essenciais para a continuidade e o sucesso dos programas de imunização ${ }^{14}$. Em estudo realizado por Geronutti et al. 25 com idosos atendidos em um Centro de Saúde Escola em Botucatu (São Paulo), a mídia (rádio e televisão) figurou como um dos importantes meios de divulgação das campanhas de vacinação contra Influenza.

Conclui-se que é fundamental a adoção de estratégias de atenção à saúde com a finalidade de contribuir para o sucesso dos programas de imunização, garantindo a plenitude da cobertura vacinal na população idosa. As campanhas de vacinação contra a gripe devem divulgar e esclarecer melhor os benefícios de se vacinar a partir dos 60 anos de idade, informando sobre indicações e riscos, visando à faixa etária mais jovem (60-69 anos) e voltadas especialmente aos idosos de baixa renda e aos portadores de doenças crônicas (cardiovasculares, pulmonares, metabólicas) para ampliar a cobertura vacinal nestes grupos específicos que vêm apresentando baixa adesão às campanhas.

O maior envolvimento dos profissionais de saúde responsabilizando-se por orientações mais claras para essa população é indispensável. Sugere-se uma participação mais ativa por parte das equipes do ESF, tanto em âmbito domiciliar como em locais oportunos para a divulgação, tais como centros comunitários, comércio local, atividades de bairro e igrejas. 


\section{Resumo}

Este trabalho teve como objetivo verificar a cobertura vacinal contra a gripe em idosos e a associação entre a vacinação e variáveis socioeconômicas, demográficas, de saúde e estilo de vida. Estudo de natureza epidemiológico-descritiva, transversal, realizado de abril de 2008 a setembro de 2009, com 990 idosos do Município de Cambé, Paraná, Brasil. A cobertura vacinal foi de 74,6\%. A maior taxa de adesão foi entre os indivíduos com idades de 70-79 anos [razão de prevalência ajustada $(R P)=1,05]$ e 80 anos ou mais $(R P=1,03)$, hipertensos, e com renda mensal de até 3 salários mínimos $(R P=1,10)$ ou superior $(R P=1,17)$ comparados aos idosos sem renda. Idosos sedentários $(R P=0,96)$ aderiram menos à vacinação, e divorciados/separados $(R P=0,92)$ apresentaram menor adesão comparados aos casados. Os resultados sugerem a necessidade de estratégias de atenção à saúde, considerando os fatores que interferem na adesão voluntária à vacinação, contribuindo para aumentar as chances de sucesso dos programas de imunização.

Vacinas Contra Influenza; Programas de Imunização; Saúde do Idoso

\section{Colaboradores}

E. C. Campos realizou a proposta do artigo, delineou o estudo, coordenou a coleta e tabulação de dados, realizou as análises dos dados e redação do texto. L. C. P. Sudan contribuiu na revisão da literatura, na revisão da análise dos dados e na redação do texto. E. D. Mattos auxiliou no delineamento do estudo, no planejamento da coleta, na análise dos dados e contribuiu na revisão da literatura. R. Fidelis realizou a análise estatística dos dados, colaborou na confecção de tabelas, na apresentação e discussão dos resultados e na redação do texto.

\section{Agradecimentos}

À Secretaria Municipal de Saúde de Cambé e aos profissionais enfermeiros e agentes comunitários de saúde das unidades básicas de saúde.

\section{Referências}

1. Paskulin LMG, Vianna LAC. Perfil sociodemográfico e condições de saúde auto-referidas de idosos de Porto Alegre. Rev Saúde Pública 2007; 41:757-69.

2. Campagna AS, Dourado I, Duarte EC, Daufenbach LZ. Mortalidade por causas relacionadas à influenza em idosos no Brasil, 1992 a 2005. Epidemiol Serv Saúde 2009; 18:209-18.

3. Lima-Costa MF. Fatores associados à vacinação contra gripe em idosos na região metropolitana de Belo Horizonte. Rev Saúde Pública 2008; 42:100-7.

4. Reis PO, Nozawa MR. Análise do programa de vacinação de idosos de Campinas, SP. Ciênc Saúde Coletiva 2007; 12:1353-61.

5. Dip RM. Vacinação contra gripe em idosos não institucionalizados: estudo de base populacional [Dissertação de Mestrado]. Londrina: Universidade Estadual de Londrina; 2007.

6. Portal Saúde. Ministério anuncia estratégia de vacinação contra a gripe. http://portal.saude.gov.br/ portal/aplicacoes $/$ noticias $/$ default.cfm?pg=dsp DetalheNoticia\&id_area $=1498 \& C O \_N O T I C I A=$ 12304 (acessado em 29/Mar/2011).
7. Fundação Nacional de Saúde. Campanha Nacional de Vacinação do Idoso 2002: informe técnico. Brasília: Fundação Nacional de Saúde; 2002.

8. Portal Saúde. Campanhas da saúde: vacinação contra a gripe - 2009. Disponível em: http://portal. saude.gov.br/ portal/saude/visualizar_texto.cfm? idtxt=33676\&janela=1 (acessado em 29/Mar/2011).

9. Francisco PMSB, Donalisio MRC, Latorre MRD. O impacto da vacinação contra influenza na mortalidade por doenças respiratórias em idosos. Rev Saúde Pública 2005; 39:75-81.

10. Fiore AE, Uyeki TM, Broder K, Finelli L, Euler GL, Singleton JA, et al. Prevention and control of influenza with vaccines: recommendations of the Advisory Committee on Immunization Practices (ACIP), 2010. MMWR Recomm Rep 2010; 59:1-62.

11. Donalisio MR. Política brasileira de vacinação contra a influenza e seu impacto sobre a saúde do idoso. Cad Saúde Pública 2007; 23:494-5.

12. Dodet B. Immunity in the elderly. Vaccine 2000; 18:1565. 
13. Donalisio MR, Ruiz T, Cordeiro R. Fatores associados à vacinação contra influenza em idosos em município do Sudeste do Brasil. Rev Saúde Pública 2006; 40:115-9.

14. Francisco PMSB, Barros MBA, Cordeiro MRD. Vacinação contra influenza em idosos: prevalência, fatores associados e motivos da não-adesão em Campinas, São Paulo, Brasil. Cad Saúde Pública 2011; 27:417-26.

15. Francisco PMSB, Donalisio MRC, Barros MBA, César CLG, Carandina L, Goldbaum M. Fatores associados à vacinação contra a influenza em idosos. Rev Panam Salud Pública 2006; 19:259-64.

16. Francisco PMSB, Donalisio MRC, Barros MBA, César CLG, Carandina L, Goldbaum M. Vacinação contra influenza em idosos por área de residência: prevalência e fatores associados. Rev Bras Epidemiol 2006; 9:162-71.

17. Burns VE, Ring C, Carroll D. Factors influencing vaccination uptake in an elderly, communitybased sample. Vaccine 2005; 23:3604-8.

18. Moura M, Silva LJ. Pesquisa de opinião sobre as campanhas de vacinação contra a influenza no Estado de São Paulo. Boletim Epidemiológico Paulista $2004 ; 1: 8-10$.

19. Sarriá A, Timoner J. Determinants de la vacinación de la gripe en personas mayors de 65 anos. Rev Esp Salud Pública 2002; 76:17-26.

20. Mac Donald R, Baken L, Nelson A, Nichol, KL. Validation of self-report of influenza and pneumococcal vaccionation status in elderly outpatients. Am J Prev Med. 1999; 16:173-7.

21. Barros AJ, Hirakata VN. Alternatives for logistic regression in cross-sectional studies: an empirical comparison of models that directly estimate the prevalence ratio. BMC Med Res Methodol 2003; 3:21.

22. Zou G. A modified Poisson regression approach to prospective studies with binary data. Am J Epidemiol 2004; 159:702-6.

23. Coordenação Geral do Programa Nacional de Imunizações, Departamento de Vigilância Epidemiológica, Secretaria de Vigilância Sanitária em Saúde, Ministério da Saúde. Nova técnica no ${ }^{\circ}$ 26/08/ CGPNI/DEVEP/SVS/MS. http://www.portal.sau de.gov.br/portal/.../pdf/nota_tecnica_vacina_ido so2008.pdf (acessado em 20/Dez/2010).
24. Dannetun E, Tegnell A, Normann B, Garpenholt O, Giesecke J. Influenza vaccine coverage and reasons for non vaccination in sample of people above 65 years of age, in Sweden, 1998-2000. Scand J Infect Dis 2003; 35:2074-8.

25. Geronutti DA, Molina AC, Lima SAM. Vacinação de idosos contra a Influenza em um Centro de Saúde Escola do interior do Estado de São Paulo. Texto \& Contexto Enferm 2008; 17:336-41.

26. O'Malley AS, Forrest CB. Immunization disparities in older Americans: determinants and future research needs. Am J Prev Med 2006; 31:150-7.

27. Gomes R, Nascimento EF, Araújo FC. Por que os homens buscam menos os serviços de saúde do que as mulheres? As explicações de homens com baixa escolaridade e homens com ensino superior. Cad Saúde Pública 2007; 23:565-74.

28. Albano BR, Basílio MC, Neves JB. Desafios para a inclusão dos homens nos serviços de atenção primária à saúde. Revista Enfermagem Integrada 2010; 3:554-63.

29. Departamento de Ações Programáticas Estratégicas, Secretaria de Atenção à Saúde, Ministério da Saúde. Nota técnica no ${ }^{\circ}$ 07/2009. Política nacional de atenção integral à saúde do homem - princípios e diretrizes. http://www.conass.org.br/arquivos/ file/nt_072009.pdf (acessado em 18/Out/2011).

30. Vilarino MAM, Lopes MJM, Bueno ALM, Brito MRV. Idosos vacinados e não vacinados contra a influenza: morbidade relatada e aspectos sociodemográficos, Porto Alegre (RS, Brasil), 2004. Ciênc Saúde Coletiva 2010; 15:2879-86.

31. Mangtani P, Breeze E, Stirling S, Hanciles S, Kovats S, Fletcher A. Cross-sectional survey of older peoples' views related to influenza vaccine uptake. BMC Public Health 2006; 6:249.

32. Landim FLP, Opaleye MGC, Varela ZMV. Promoção humana para o autocuidado em saúde na família. Família, Saúde e Desenvolvimento 2000; 2:55-65.

33. Dip RM, Cabrera AS. Vacinação contra a gripe como estratégia de promoção de saúde em idosos. Geriatria \& Gerontologia 2008; 2:81-5.

34. Burns VE, Ring C, Carroll D. Factors influencing vaccination uptake in an elderly, communitybased sample. Vaccine 2005; 23:3604-8.

Recebido em 21/Jun/2011

Versão final reapresentada em 21/Dez/2011 Aprovado em 26/Jan/2012 